\title{
miR-144/451 in hematopoiesis and beyond
}

Ting Wang ${ }^{1}$, Fan $W^{1}$ and Duonan $\mathrm{Yu}^{1,2,3^{*}}$

\begin{abstract}
MicroRNAs (miRNAs), a class of 18-25 nucleotide (nt) non-coding RNAs, usually inhibit the expression of their target genes. They are transcribed from endogenous genes and are processed for maturation by multiple pathways. miR144/451, a bicistronic gene locus, encodes miR-144 and miR-451, both of which are highly conserved in evolution. These two miRNAs are on the same primary RNA molecule whose transcription is controlled by multiple nuclear proteins, including GATA1, GATA4, Myc, Oct1, Pax4, FXR, AP1, SMAD3 and SMAD4 depending on tissue types. They are abundant and almost exclusively exist in red blood cells, but low expression of both miR-144 and miR-451 are also detected in non-erythroid lineages. Interestingly, deletion of both miR-144 and miR-451 DNA sequences coding pre-miR-144/451 hairpins in mice leads only mild microcytic anemia but worsen upon a number of stresses including developmental stress, acute blood loss, phenohydrazine-induced hemolysis and precursor depletion by chemotherapeutic drug 5-FU. Such knockout animals older than 15 months also spontaneously develop malignant tumors including B-lymphoma and acute myeloid leukemia, indicating that miR-144/451 is a bona fide tumor-suppressing gene in non-erythroid cells, though its levels are much lower compared to that in red blood cells. Consistent with the findings in animals, disruption of miR-144/451 expression and their abnormal functions are observed in human hematopoietic and non-hematopoietic organs. Moreover, miR-451 is the only miRNA discovered so far whose maturation does not depend on Dicer, an enzyme required by all other miRNAs for maturation. This review focuses on the biogenesis, transcriptional regulation and biological roles of miR-144/451 in erythropoiesis, tumor initiation and other pathological conditions.
\end{abstract}

Keywords: miR-451, miR-144, Biogenesis, Erythropoiesis, Cancer, Transcription

\section{Background}

microRNA (miRNA) is a class of endogenous non-protein-coding RNAs, only $18-25 \mathrm{nt}$ in length, and was first discovered in Caenorhabditis elegans by Ambros' group [1]. Hundreds of miRNAs have been confirmed functional in mammals, and thousands of others are still with unknown functions. miRNAs are expected to host gene regulation in virtually all mammalian tissues and cells [2]. miRNAs are generated typically as monocistronic or polycistronic gene loci (the latter is about 50\% [3]). These miRNA gene loci usually exist as independent units, but can be in introns of protein-coding genes as well (Fig. 1). Activating signals drive RNA polymerase II (Pol II) to transcribe miRNA genes into large primary hairpin-containing transcripts (pri-miRNAs) [4]. Pri-miRNAs

\footnotetext{
* Correspondence: dnyu@yzu.edu.cn

${ }^{1}$ Jiangsu Key Laboratory of Experimental \& Translational Non-coding RNA Research, Yangzhou University School of Medicine, Yangzhou, China

${ }^{2}$ Institute of Translational Medicine, Yangzhou University School of Medicine, Yangzhou, China

Full list of author information is available at the end of the article
}

are processed in nucleus through the canonical pathway (Fig. 1) [5, 6] and the resultant hairpin precursors (pre-miRNA, usually 60-70 nt long) are further processed by RNase III enzyme Dicer $[7,8]$ to generate a miRNA duplex. miRNA duplex is then incorporated into a Argonaute-containing, RNA-induced silencing complex (RISC), where the functional mature miRNAs are produced. Besides the canonical pathway, other alternative pathways have been described for mature miRNA generation [9-13] (Fig. 1). Noncanonical pathways also produce miRNAs by processing endogenous short-hairpin RNAs (shRNAs) and chimeric hairpin RNAs (produced in tandem with or as part of small nucleolar RNAs, tRNA-like molecules, and small nuclear RNAs) [2]. Regardless of how miRNAs are generated, mammalian miRNAs typically inhibit large networks of downstream gene targets primarily through the Watson-Crick base-pairing of miRNA "seed" sequences to the specific binding sites in target RNAs, which leads to deadenylation, 


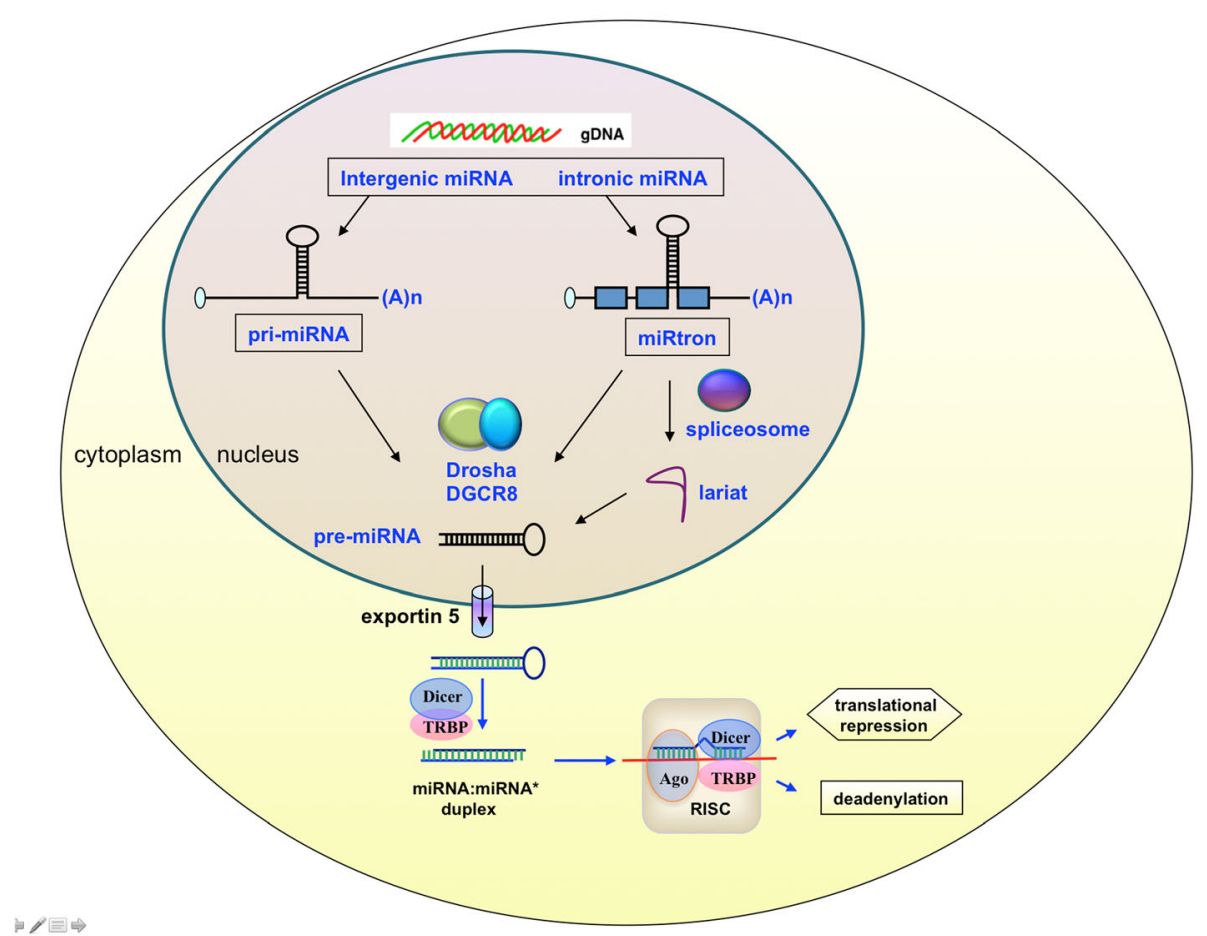

Fig. 1 miRNAs are encoded by genes located either independently (intergenic) or within introns of protein-coding genes (intronic). miRNA genes are transcribed by RNA polymerase II into primary miRNA (pri-miRNA), usually with 5' cap and 3' poly A structures. RNase III enzyme Drosha, together with its partner DGCR8 protein, cleaves pri-miRNAs to produce the 60-70 nt long hairpin precursors (pre-miRNA) through canonical pathway. Pri-miRNAs from intronic miRNA genes are also called miRtrons. miRtrons can be spliced to lariat structures by spliceosome and further processed to pre-miRNAs. The pre-miRNAs in nuclei are exported into the cytoplasm by exportin-5 protein and cleaved to short miRNA duplexes by RNase III enzyme Dicer. One strand (passenger strand or miRNA*) is expulsed and the other strand (guide strand, i.e., mature miRNA) is bound to Ago2 in RNA-induced silencing complex (RISC). Mature miRNA, primarily through the "seed" region (miRNA nucleotides 2-8), binds to specific sequences within target RNAs, typically within the 3' untranslated region (UTR) of the protein coding RNAs or other transcripts to inhibit the expression of the targets to which they bind

RNA decay, and/or translational suppression [14]. The details about miRNA classification, biogenesis, function and target identification can be found in recent reviews $[2,15]$.

miR-144/451 is a bicistronic gene locus on chromosome 17 and chromosome 11 in human and mouse genomes, respectively. The miR-144/451 locus encodes two highly conserved miRNAs: miR-144-3p and miR-451a (unless otherwise indicated, miR-144 and miR-451 referred in this review are miR-144-3p and miR-451a, respectively) [16]. miR-144 and miR-451 are highly expressed in erythroid cells $[16,17]$, and knockout of miR-144/451 sequences that contain pre-miR-144/451 hairpins in mice disrupts erythroid homeostasis [18-20]. Interestingly, recent in vivo evidence reveals that miR-144/451 alone acts as a weak tumor suppressive miRNA locus that inhibits B-lymphomagenesis and tumorigenesis of myeloid lineage cells in mice [21], clearly indicating that miR-144/451 also functions in non-erythroid cells, though its levels are much lower than that in red blood cells. Moreover, miR-451, but not miR-144, is the only miRNA whose maturation is Dicer-independent [22-24]. Still, a lot about
miR-144/451 biology is yet to be defined. In this review, we focus on recent miR-144/451 studies to elucidate the biogenesis, transcriptional regulation and biological functions of miR-144/451 in erythropoiesis, cancer development and other pathological conditions. We believe that genetic and pharmacologic manipulations that change miR-144/451 levels could impact the development courses of various diseases.

\section{Regulation of miR-144/451 expression at transcriptional levels}

miR-144 and miR-451 are encoded by a single miRNA locus and their mature sequences, only $100 \mathrm{bp}$ apart, are on the same primary RNA [16]. The miR-451 expression is largely restricted to red blood cells, though a low level of miR-451 is observed in non-erythroid lineages. Remarkably, about half of the miRNAs in mouse fetal liver (FL) erythroblasts are miR-451 [16, 17], while miR-144 levels are much lower than those of miR-451 in red cells $[16,20]$. In addition, miR-144 expression is relatively more ubiquitous than the expression of miR-451 during embryonic development [25, 26], suggesting that not 
only overlapping but also independent mechanisms regulate the levels of functional miR-144 and miR-451. Nevertheless, at least eight nuclear factors are involved in directly regulating the expression of miR-144/451 (Table 1) in various tissues. Whether the expression of miR-144/451 is activated or inhibited depends on the transcription factors and also probably cell types.

miR-144/451 expression is strongly induced upon differentiation of erythroid cells in zebrafish, mice, and humans [27-29]. Chromatin immunoprecipitation (ChIP) and gene promoter activity assays demonstrate that GATA1, a nuclear factor that potentially regulates more than 1 thousand of genes in erythroblasts [30,31], binds the gene promoter region at $\sim 2.8 \mathrm{~kb}$ from the transcription initiation site of the miR-144/451 locus and activates miR-144/451 transcription in erythroid cells [16].

miR-144/451 is strongly expressed during red cell development. However, numerous studies have shown disrupted levels of miR-144/451 in non-erythroid tumor cells [32-38], including diffuse large B-cell lymphomas (DLBCL) [21]. In many DLBCLs, the level of Myc, a protooncogenic protein that directly targets an estimate $15 \%$ of human genes, is altered [39]. Myc can directly inhibit a large set of miRNAs in B-lymphoma cells in a consensus "E-box" sequence-independent manner [40]. Consistent with this work, Myc can occupy the conserved DNA sequence located within the miR-144/451 gene promoter and directly repress the expression of miR-144/451 [21]. This explains why high MYC-expressing human and murine B-lymphoma cells usually show low abundance of miR-144 and miR-451 compared with normal B-lymphocytes.

Transcription factor paired box gene 4 (PAX4) binds to specific DNA sequence [41]. PAX4 is one of the PAX gene family members and its abnormal expression has been shown in many malignancies, including breast adenocarcinomas and neck squamous cell carcinomas [42]. Interestingly, ChIP and a promoter reporter assay demonstrate that PAX4, similar to Myc, transcriptionally inhibits the expression of miR-144 and miR-451, and thus upregulating miR-144/451 target genes ADAMTS5 and ADAM10, two A disintegrin and metalloproteinase (ADAM) family proteins, both of which can promote cancer invasion and metastasis [42].

The fourth transcription factor that occupies the miR-144/451 promoter region is GATA4 [43]. Same as GATA1, GATA4 belongs to the GATA gene family that has six GATA members. Whereas GATA1, GATA2 and GATA3 are mainly expressed in blood cells, GATA4, GATA5 and GATA6 are usually expressed in cardiomyocytes and endodermal cells [44]. It has been demonstrated that GATA4 binds to two GATA sites in the miR-144/451 promoter sequence (at positions -512 and - 826) in cardiomyocytes. Interestingly, GATA4 activated miR-144 and miR-451 coordinate the survival of cardiomyocytes [43].

Transcription factor OCT1 (POU2F1) also transcriptionally activates the miR-451 expression in glioblastoma multiforme (GBM) cells. In this case, metabolic stress, such as low glucose conditions, activates AMP-activated kinase (AMPK), which represses the activity of OCT1 protein by phosphorylation at serine 335 . OCT1 activates miR-144/451 transcription by occupying multiple sites in the promoter region, and shutdown of OCT1 activity by AMPK reduces miR-451 expression, which further increases AMPK activity through the upregulation of miR-451 target gene Cab39 [45]. Thus, in GBM cells, metabolic stress triggers a Cab39/AMPK-mediated positive feedback response between miR-451 and OCT1, which allows tumor cells to quickly adapt to variations of glucose concentrations in the tumor microenvironment. These findings in GBM cells uncover miR-451 as a major player in AMPK signaling, which is consistent with a recent finding in erythroid cells that miR-451 is an important effector that represses Cab39/AMPK activity [46]. The inconsistency is that deficiency of miR-451 in nucleated erythroid cells results in apoptosis, rather than adaptation for survival, in numerous stress conditions, including deprivation of glucose in culture medium ([46]; unpublished data).

Table 1 Transcription factors that regulate miR-144/451 expression

\begin{tabular}{|c|c|c|c|c|}
\hline $\mathrm{TF}$ & Activity & Region ( bp) & Target Cell & Reference \\
\hline$\overline{\text { GATA1 }}$ & Activation & -2800 & Erythroid cell & [16] \\
\hline Myc & Repression & -400 & B-lymphoma cell & [21] \\
\hline PAX4 & Repression & -2000 & Breast cancer cell & [42] \\
\hline GATA4 & Activation & $\begin{array}{l}-512 \\
-826\end{array}$ & Cardiomyocyte & [43] \\
\hline OCT1 & Activation & $-6600 \sim 8200$ & Glioblastoma cell & [45] \\
\hline FXR & Activation & - 3000 (human) -12,000 (mouse) & Hepatocyte & [47] \\
\hline AP1 & Activation & -1000 & Neuron & [51] \\
\hline SMAD3/4 & Activation & -1000 & Glioblastoma cell & [52] \\
\hline
\end{tabular}


Farnesoid X Receptor (FXR) is a nuclear receptor and controls many aspects of lipid metabolism. FXR binds response elements in the upstream sequence of miR-144/451 locus to activate miR-144/451 transcription, which results in high levels of miR-144/451 in hepatocytes followed by inhibition of miR-144 target, hepatic ATP binding cassette transporter A1 (ABCA1) [47]. ABCA1 is a positive regulator of plasma high-density lipoprotein (HDL) levels and decrease of ABCA1 reduces plasma HDL levels. FXR/miR-144 thus lowers plasma HDL that is considered cardioprotective [48].

In addition to GATA1, Myc, PAX4, GATA4, OCT1 and FXR, activator protein-1 (AP-1) also transcriptionally activates miR-144/451. ADAM10 can reduce the production of amyloid $\beta$-peptide $(A \beta)$, the essential protein that causes Alzheimer's disease (AD). However, the ADAM10 expression is repressed in AD [49]. Interestingly, miR-144 is one of the few miRNAs that is consistently increased in the brain tissues of elderly people and AD patients [50], suggesting that high levels of miR-144 might be detrimental to neurons in brain tissues. Notably, $\mathrm{A} \beta$ activates $\mathrm{AP}-1$, and the latter binds to the recognition sites in the miR-144/451 promoter and transcribes miR-144/451, both of the miRNAs are capable of inhibiting the expression of the ADAM10 protein [51]. Thus, AP1 transcribed miR-144/451 is implicated in $\mathrm{AD}$ pathogenesis by direct downregulation of neuron protective ADAM10.

Both SMAD3 and SMAD4 reduce the growth of GBM A172 cells in vitro [52], and the inhibitory effect might be due to the enhanced miR-451 transcription. miR-144/ 451 promoter region ( $1135 \mathrm{bp}$ from the miR-451 mature sequence) has been identified to contain binding elements for SMAD3 and SMAD4, with a 157 bp interval. Promoter activity assay reveals enhanced reporter activity in the presence of SMAD3 or/and SMAD4 in multiple glioma cell lines, suggesting that miR-451 expression is transcriptionally activated by SMAD3 and SMAD4 (52).

In summary, GATA1, Myc, PAX4, GATA4, OCT1, FXR, AP-1 and SMAD so far have been identified as transcription factors that occupy the promoters of the miR-144/451 locus to either activate or inhibit the expression of miR-144 and miR-451 (Fig. 2).

\section{miR-144/451 in erythrocyte development and red cell diseases}

The levels of miR-144 and miR-451 are very abundant in erythroid cells $[16,29,53,54]$. Over expression of miR-144 or miR-451 enhances maturation of murine erythroleukemic cells, whereas depletion of miR-451 has the opposite effect $[29,55]$. Surprisingly, in vivo deletion of such abundantly expressed miR-144/451 genes only leads to mild hemolytic anemia [18-20, 46], partially due to the erythrocytes' impaired capacity to remove reactive oxygen species (ROS) [19]. In animals, deletion of genomic miR-144/451 sequences elevates the level of miR-451 target gene (Ywhaz) product 14-3-3zeta, which sequesters the nuclear factor FoxO3 in the cytoplasm, thereby blocking transcription of anti-oxidant enzymes catalase (Cat) and glutathione peroxidase 1 (Gpx1) by FoxO3 [19]. This mechanism explains the hypersensitivity of miR-144/451 gene knockout erythrocytes to oxidants, since anemia is much worse upon oxidative stress generated by treating zebrafish with 1-phenyl-2-thiourea (PTU) $[16,19]$ and by treating mice with phenohydrazine (PHZ) [19, 46] or mature red blood cells with hydrogen peroxide (H2O2). Both PTU and PHZ are strong oxidants that induce rapid hemolysis of red cells.

A recent study demonstrates that erythroblasts isolated from miR-144/451 knockout mouse fetal liver or adult bone marrow and spleen exhibit more cell death during recovery from acute anemia, including PHZ-induced rapid destruction of erythrocytes, 5-fluorouracil (5-FU)-induced elimination of erythroid precursors, as well as acute blood loss by bleeding, suggesting that miR-144/451 is a main protector of erythroid cells in pathological conditions associated with not only oxidant stress but also other dramatically increased erythropoietic demand [46]. This effect is mediated by derepression of Cab39, a direct miR-451 target, followed by activation of the downstream effectors LKB1/AMPK. Interestingly, this anti-apoptotic effect of miR-451 is tumor suppressor p53-dependent. Ablation of p53 blocks the apoptosis of erythroblasts in miR-144/451 knockout animals [46]. However, miR-144/451 depletion via expression of decoy RNAs in adult mice [55] seems to inhibit erythropoiesis more severely than targeted deletion of the gene locus. This discrepancy is likely due to technical and/or biological differences between the two experimental approaches and illustrates the importance of using multiple modalities to assess miRNA function.

At least three groups have independently examined the effects of knockdown of miR-451 in zebrafish by injecting antisense morpholino (MO). Dore and $\mathrm{Du}$ et al. reported that treatment with anti-miR-451 MO caused severe anemia [16,56], while Pase et al. found much milder erythroid phenotype with a similar treatment [28]. This discrepancy was not explained until Yu et al. demonstrated that deficiency of miR-451 expression in mice interferes FoxO3-mediated anti-oxidant signaling [19]. PTU is a melanin synthesis inhibitor that is used in Dore and Du's experiments to improve the visualization of zebrafish embryos by reducing the melanin in embryonic tissues. However, PTU is an oxidant that oxidizes hemoglobin in the embryos and thus synergizes the deleterious effect of miR-144/451 deficiency in zebrafish [19]. The enhanced loss of hemoglobin due to 


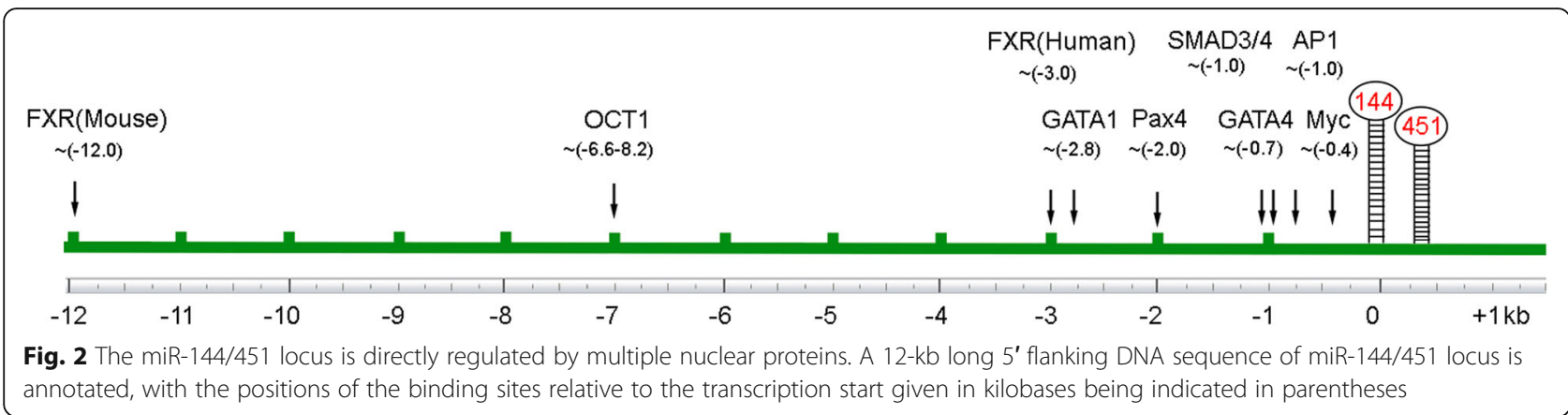

PTU treatment confirms the anti-oxidantive function of miR-451 in erythroid cells and also demonstrates that this function is well conserved in evolution.

miR-144 is coexpressed with miR-451 in a bicistronic primary transcript whose synthesis is directly activated by transcription factor GATA1 in erythroblasts [16]. However, the sequences of these two miRNAs are entirely different. Nevertheless, enforced expression of either miR-144 or miR-451 enhances maturation of murine erythroleukemic cells $[29,55]$, whereas depletion of either miR-144 or miR-451 using lentiviral-driven 'decoy' RNAs inhibits the maturation of hematopoietic stem/ progenitor cells into mature erythroblasts [55]. More interestingly, miR-144 and miR-451 depletion additively inhibits erythropoiesis, suggesting the similar biological effects of miR-144 and miR-451, despite their different sequences, especially "seed" sequences that mediate the inhibition of distinct sets of target mRNAs [55]. However, this phenomenon has not yet been validated in gene knockout animals.

miR-144 also negatively regulates the expression of globin genes in zebrafish by targeting klfd, a Kruppellike transcription factor that drives the transcription of embryonic alpha-globin. Specifically, miR-144 deficiency directly derepresses klfd expression, thereby promoting synthesis of embryonic alpha-globin [27, 56]. Interestingly, klfd inversely transactivates miR-144/451 expression in zebrafish, defining a feedback loop that appears to fine-regulate embryonic alpha-globin levels during erythropoiesis. Whether this regulatory axis also exists in mammalian erythropoiesis is unknown. A recent study demonstrates that high levels of miR-144 correlate with attenuated anti-oxidant capacity and thus more severe anemia in homozygous sickle cell disease (HbSS) erythrocytes [57]. This observation is certainly inconsistent with the findings that enforced expression of miR-144 enhances maturation of murine erythroleukemic cells [29], suggesting that miR-144 or miR-451 may function differently under different physiological and pathological conditions.

High miR-451 level is associated to the resistance of Malaria infection [58]. Malaria is one of the most prevalent diseases in tropical and subtropical areas. Five species of the Plasmodium parasite can cause human malaria. World Health Organization (WHO) has reported that $99 \%$ of deaths in human malaria is caused by Plasmodium falciparum (P. falciparum) (World Malaria Report 2015, WHO). Human hemoglobin S (HbS) is a common hemoglobin variant in endemic areas of malaria and red blood cells containing $\mathrm{HbS}$ are confirmed resistant to malaria infection. Increased phagocytosis of sickle cells $[59,60]$ and decreased parasite invasion and growth [61] are among the factors that attribute to the resistance. Recent reports demonstrate that miR-451 is not only highly enriched in HbSS red cells [62] but also accumulated within the $P$. falciparum parasite $[58,63]$. Moreover, high levels of miR-451 in sick erythrocytes contribute to malaria resistance. Specifically, high miR-451 levels in sickle red cells dramatically reduces the growth of $P$. falciparum through an unique non-canonical pathway in which miR-451 from host red cells enters $P$. falciparum and covalently forms a fusion transcript with mRNA of a subunit of the cAMPdependent Protein Kinase (PKA-R) in parasites. The fused RNA impairs the translation of $P$. falciparum PKA-R, an essential enzyme for parasite growth [58]. These findings demonstrate that sickle erythrocytes can transfer their enriched miR-451 into parasites to suppress malaria infection. These findings, together with a recent report that dietary miR-451 (presumably from fish powder) is capable of passing through the gastrointestinal system into circulating blood to regulate the erythroid functions of mice [64], support the notion that miRNAs may perform cross-species gene regulation [65].

\section{miR-144/451 in tumorigenesis}

The levels of miR-144 or miR-451 in non-erythroid cells is very low; however, many studies have reported the deregulation of their expression and abnormal functions in various malignancies (Table 2). These two miRNAs participate in tumorigenesis with either oncogenic or tumor suppressive functions. The tumors associated with disrupted miR-144 or miR-451 expression and functions 
Table 2 The target genes and functions of miR-144 and miR-451 in tumorigenesis

\begin{tabular}{|c|c|c|c|c|}
\hline miRNA & Tumor type & Target & Function & Reference \\
\hline miR-451 & B-cell lymphoma & c-Myc & suppressive & [21] \\
\hline miR-144 & B-cell lymphoma & BCL6 & suppressive & [37] \\
\hline $\operatorname{miR}-451$ & T cell acute lymphoblastic leukemia & c-Myc & suppressive & [34] \\
\hline miR-451 & multiple myeloma & TSC1 & suppressive & {$[35]$} \\
\hline $\operatorname{miR}-451$ & lung cancer & c-Myc & suppressive & [66] \\
\hline miR-451 & lung cancer & RAB14 & suppressive & [67] \\
\hline miR-451 & lung cancer & Akt & suppressive & [68] \\
\hline miR-451 & lung cancer & c-Myc & suppressive & [32] \\
\hline miR-451 & glioblastoma & c-Myc & suppressive & [69] \\
\hline miR-451 & glioblastoma & Cab39 & Conditional switching control & [70] \\
\hline miR-451 & glioblastoma & Akt1 & suppressive & [71] \\
\hline $\operatorname{miR}-451$ & glioblastoma & $N / A^{a}$ & suppressive & [52] \\
\hline miR-451 & breast cancer & $14-3-3 \zeta$ & suppressive & [33] \\
\hline miR-451 & breast cancer & MDR1 & & [72] \\
\hline miR-144 & colorectal cancer & mTOR & suppressive & [73] \\
\hline miR-451 & colorectal cancer & MIF & suppressive & [74] \\
\hline miR-451 & colorectal cancer & MIF & suppressive & [75] \\
\hline miR-451 & colorectal cancer & $14-3-3 \zeta$ & suppressive & [76] \\
\hline miR-144 & nasopharyngeal carcinoma & PTEN & oncogenic & [77] \\
\hline miR-451 & nasopharyngeal carcinoma & MIF & suppressive & [78] \\
\hline miR-144 & pancreatic carcinoma & Notch-1 & suppressive & [79] \\
\hline miR-144 & insulinoma & PTEN & oncogenic & {$[80]$} \\
\hline miR-451 & liposarcoma & & suppressive & [81] \\
\hline miR-144/miR-451 & breast cancer, head and neck carcinomas & $\begin{array}{l}\text { ADAMs } \\
\text { ADAMs }\end{array}$ & suppressive (invasion \& metastasis) & $\begin{array}{l}{[42]} \\
{[42]}\end{array}$ \\
\hline miR-451 & ovarian and cervix cancers & MDR1 & oncogenic & [82] \\
\hline
\end{tabular}

${ }^{\mathrm{a}}$ Not available

include hematopoietic malignancies [21, 34, 35, 37], lung cancer [32, 66-68], glioblastoma [52, 69-71], breast cancer [33, 72], colorectal cancer [73-76], gastric cancer [74], nasopharyngeal cancer [77, 78], pancreatic cancer $[79,80]$, and other tumors $[42,81,82]$. However, no in vivo studies had confirmed such observations until a recent in vivo study demonstrated that the deficiency of miR-144/451 gene expression is a driver of B-lymphomagenesis though the tumor suppressive activity is weak by miR-144/451 alone [21]. Specifically, knockout of miR-144/451 locus initiates development of B-cell lymphoma and acute myeloid leukemia in aged mice with low incidence. The underlining mechanism is that the silenced oncogene Myc in normal hematopoietic cells is derepressed by the loss of miR-144/451 expression. Moreover, over-expressed Myc further maintains its own level by direct down regulation of miR-144/451, forming a miRNA-Myc feedback that is disrupted in B-cell lymphomas [21]. These data are consistent with the previous in vitro or ex-in vivo findings that miR-144/451 are capable of inhibiting Myc expression [32, 34, 66]. The question remains whether such weak miRNAs could be a strong enhancer if other gene mutations exist. Inactivation of tumor suppressive genes and activation of pro-proliferative genes are usually required for rapid onset and high penetrance of many cancers [39]. Thus, it will be interesting to study whether loss of miR-144/451 sensitizes the deficiency of other gene expression for cancer development.

miR-144 and miR-451 as tumor biomarkers have been widely investigated. For example, miR-451 level is much lower in cancers from the digestive system [74, 83], non-small cell lung carcinoma (NSCLC) tissues $[67,68]$, and DLBCL [21]. While majority of studies indicate miR-144 or miR-451 as tumor suppressive miRNAs, several groups suggest that miR-144 or miR-451 inherits oncogenic activities [77, 80], which is consistent with the prognosis studies that glioblastoma patients with high miR-451 levels in tumor cells have significantly short 
survival time [70] whereas patients with gastric cancer expressing lower miR-451 are associated with better outcomes [84]. These contradictory results imply that miR-451 may be a disease states-specific and tissue-specific tumor marker of diagnostic value. However, red blood cell contamination could be another explanation for these contradictory observations. Red blood cells express such high levels of miR-451 that miR-451 expression in non-erythroid cells is almost negligible. While any primary tumor is vascularized and contains large numbers of red cells, contamination of even a small number of red blood cells could affect the detection of miR-451 from cancer cells. Thus, serums may be a good source for miR-144 or miR-451 detection. Indeed, miR-451 has been investigated as diagnostic miRNA for human renal cell carcinoma [85], acute B-cell lymphoblastic leukemia [86] and gliomas [71].

Evidence has shown that miR-451 enhances the sensitivity of many types of malignancies to therapeutics. miR-451 restores the growth inhibitory effect of tamoxifen and doxorubicin in breast cancers [33, 72]. Treatment of osteosarcoma cells and NSCLC cells with miR-451 correlates with more positive response to conventional anti-cancer therapy [32, 68, 87]. However, contradictory results also have been reported that miR-451 decreases sensitivity of cancer cells to doxorubicin by increasing the levels of P-glycoprotein, the multidrug resistance 1 gene product that pumps chemotherapeutic drugs across the cell membrane to the outside of cancer cells [82]. Moreover, Decreased miR-451 expression by low glucose (a metabolic stress) inhibits cell proliferation [70, 88].

Another fundamental issue in miR-144/451 biology is that miR-144/451, especially miR-451, are considered erythroid lineage-specific genes whose expression are mainly limited to within erythrocytes and their precursors; in other words, whether low miR-144/451 expression can alter the path of normal development. A recent report has addressed this question by showing that a trace amount of miR-451 affects the clearance of ROS in red blood cells [64]. In this report, authors show that miR-144/451 gene knockout animals are capable of orally uptaking miR-451 to the blood stream from daily chow diet, presumably from fish powder in the food. Furthermore, these diet-derived miR-451 molecules, even at very low levels, can inhibit the expression of their target genes in mice. This result, together with the finding that miR-144/451 gene knockout animals develop non-erythroid malignancies including Blymphoma, acute myeloid leukemia and benign liver adenoma [21], indicates clearly that large amounts of miRNAs are not necessary for sufficient regulation of cellular functions.

\section{Function of miR-144/451 in additional tissues}

Cardiac ischemia/reperfusion (I/R)-induced injury can be strongly protected by ischemic preconditioning (IPC). Interestingly, miR-144/451 are the most up-regulated miRNAs by IPC, suggesting a positive cardioprotective role of miR-144/451 [89]. Indeed, knockout of the miR-144/451 gene locus reduces IPC-linked cardioprotection [89]. This is similar to the findings that miR-144/ 451 protects cardiomyocytes against I/R-triggered or oxidative stress-induced cell death [43]. The latter function is particularly interesting in light of the similar functions in erythroid cells, which might occur through common mechanisms. However, uptake of high-fat diet (HFD) enriches miR-451 in mouse heart and the high levels of miR-451 contribute to the HFD-induced cardiac hypertrophy, a similar pathological alteration to diabetic cardiomyopathy, the cardiac hypertrophy and impaired cardiac function in type 2 diabetes in humans. The lipotoxicity of miR-451 in cardiomyocytes can be ameliorated by cardiomyocyte-specific miR-451 gene deletion in mice by directly activating the Cab39/LKB1/AMPK signaling [90]. The apparent opposite effects of miR144/451 (alleviation of I/R-induced heart injury and promotion of HFD-induced cardiac hypertrophy) suggest that 1) miR-144 and miR-451, though produced from the same gene, might function differently; 2) the same miRNA could participate in gene regulation in a compartment-dependent or disease-dependent manner, where the latter is evidenced by our recent finding that miR-451 inhibits the same Cab39/LKB1/AMPK signaling pathway but with opposite cellular effects: protection of red cells against apoptosis [46] vs lipotoxicity in cardiomyocytes [90]. Another metabolism-related study demonstrates that HFD or diabetic condition induces the expression of hepatic miR-451 [91]. Glucose or insulin also upregulates miR-451 in hepatocytes, which leads to the inhibition of hepatic gluconeogenesis and thus low blood glucose levels [91]. Again, a negative feedback between glucose and miR-451 assures the maintenance of normal levels of blood glucose.

Construction and maintenance of epithelial cell polarity is a hallmark of normal tissue development. Disruption of cell polarity attributes to abnormal cell functions, including uncontrolled growth or progression towards malignancies [92]. One report has demonstrated that miR-451 is necessary for the development of epithelium polarity by promoting translocation of beta1 integrin to the basolateral membrane [93]. miR-451 also plays a role in autoimmune arthritis (RA). Neutrophils isolated from patients with RA contains lower miR-451 levels than those from healthy controls, and overexpression of miR-451 significantly inhibits neutrophil chemotaxis through p38 MAPK signaling and reduces the severity of arthritis in mouse RA models [94]. Erythropoietin 
(EPO), a cytokine widely used for treating chronic anemia [95], limits the generation of tissue damage molecules and thus exerts remarkable neuroprotective effects in disease models including Alzheimer's disease (AD) and Parkinson's disease (PD) [96]. Interestingly, EPO treatment downregulates miR-451 expression, which enhances EPO-mediated anti-oxidant effects [97]. This is somewhat different from the finding in erythroid cells where miR-451 helps erythroid cells with reduction of oxidant stress [19]. This discrepancy may further confirm that miRNAs function in a tissue or cell compartment-specific manner.

Like miR-451, miR-144 also involves the lipid metabolism [98]. The pathological characteristic of atherosclerosis is the accumulation of cholesterol in macrophages. Adenosine triphosphate-binding cassette transporter A1 (ABCA1) can facilitate the efflux of cholesterol to apolipoprotein A-I protein and increase HDL biogenesis in the liver to reduce cholesterol levels in macrophages. miR-144 directly inhibits hepatic ABCA1 expression both in vivo and in cultured cells, thereby attenuating cholesterol efflux from macrophages and reducing HDL levels in circulating blood [47, 98], suggesting its harmful role in the development of atherosclerosis. miR-144 also inhibits the antiviral response of animals. Ectopic expression of miR-144 in mouse lung epithelium facilitates increased replication of influenza virus, encephalomyocarditis virus and vesicular stomatitis virus [99]; ablation of miR-144/451 reduces the replication of influenza virus in the respiratory system and decreases the severity of pneumonia and bronchial damage [99]. Moreover, aberrant miR-144 expression is associated with the pathogenesis of psychiatric disorders. In fear extinction-impaired animals, over expression of miR-144 in the amygdala can successfully rescue fear extinction memory by targeting Pten, Notch1 and Spred1, a group of genes important for the control of fear extinction [100], highlighting the significance of miR-144 for the memory-related activities.

\section{Biogenesis of miR-451 is dicer-independent}

Mature miRNA generation proceeds via multiple pathways but shares a broad requirement for Dicer for trimming part of the hairpin sequence of the miRNA precursor. However, miR-451 seems to be the only miRNA whose precursors are cleaved by Ago2 rather than Dicer [22-24]. The primary transcript of miR-144/ 451 is a typical Drosha substrate, but the resultant miR-451 (but not miR-144) stem-loop precursor is too short (only $42 \mathrm{nt}$ long in mice) that Dicer cannot cleave it. The hairpin instead directly enters Ago2 protein to produce a functional miR-451 molecule. miR-451 is dominant in erythroid cells and transcriptionally regulated by the nuclear protein GATA1. However, the miR-144 as a partner is not activated to the same level as miR-451 during erythroid maturation, suggesting that non-canonical biogenesis, together with differential stability of the miRNAs, may be the reason that the levels of miR-451 and miR-144 are different in erythroid cells.

This unique miRNA biogenesis pathway needs two requirements: first, the hairpin structure must be very short. Majority of miRNAs are about 60-70 nt long [2], but the miR-451 hairpin is only $42 \mathrm{nts}$ in a diverse set of animals including humans, mice and zebrafish [101]; second, point mutations in $10 \mathrm{C}, 11 \mathrm{C}$ on $5^{\prime}$ arm and 36G, 37G on 3' arm are strongly detrimental for mature miR-451 generation and function, while unpairing at the nt $35 \mathrm{U}$ position on $3^{\prime}$ stem enhances miR-451 function

\section{セே \\ $\perp \perp$}

$5^{\prime} \mathrm{A}$

\section{AACCGUUACCAUUACUGA}

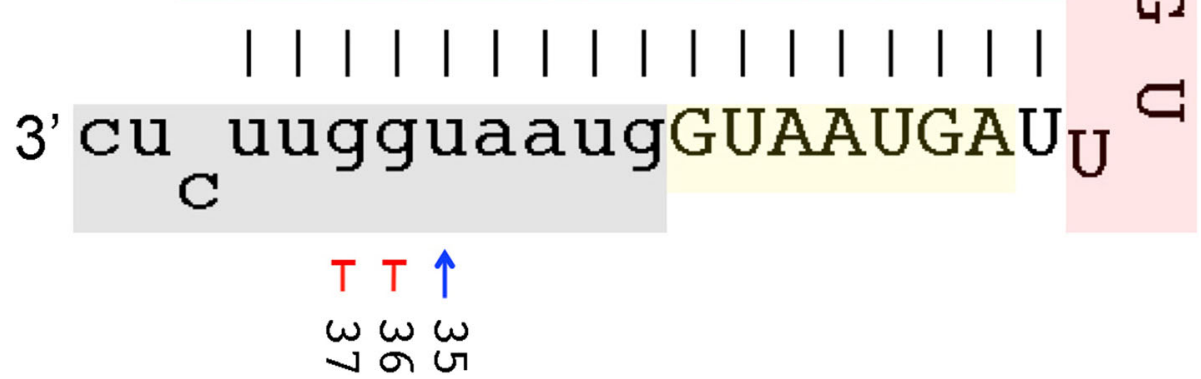

Fig. 3 Mutational analysis of miR-451 precursor. The pre-miR-451 hairpin contains the Ago2-cleaved byproduct (grey), the resected region (yellow), and the mature miR-451 (pink). The mutants are designated by their positions within the pre-miR-451 hairpin 
[24, 101], indicating that a specific base-paring in the hairpin structure is required for efficient splicing by Ago2 (Fig. 3).

Though the biogenesis of miR-451 occurs independently of Dicer and Ago2 cut the 3' stem of the miR-451 precursor, how the Ago2-cleaved intermediates (26-nt, 29-nt in length in human cells) are trimmed to the 22-nt long mature miR-451 is not clear. One recent observation reveals that poly A specific ribonuclease (PARN), an enzyme controlling the poly A tail length and mRNA stability in eukaryotes [102], is responsible for the shortening of Ago2-cleaved miR-451 precursor. Surprisingly, without shortening, the Ago2-cleaved miRNA intermediates still silence the target gene expression, indicating that once cleaved by Ago2, modification is dispensable for target silencing [103]. Ago2 belongs to a protein family that includes four Ago members, but only Ago2 is endonucleolytically active in mammalian cells [104]. Surprisingly, eIF1A as a translation initiation factor directly augments the Dicer-independent pre-miR-451 cleavage by Ago2, suggesting that eIF1A also facilitates miR-451 biogenesis [105].

\section{Future perspective}

Although strong evidence has shown the participation of miR-144/451 in erythropoiesis and tumorigenesis, our understanding of these two miRNAs during the last several years has not accelerated. Considering their pervasiveness, abundance and capability targeting diverse genes, we strongly believe that miR-144/451 must have more functions that we need but have yet to define. For example, it is commonly accepted that miRNAs are considered as "tuning" rather than "switching" genes. However, whether two different "tuning" miRNAs, especially from a single gene locus, are additive to become a "switching" gene, or whether two different "tuning" miRNAs from the same gene locus inherit completely opposite activities is still not clear. In addition, whether low levels of miR-144 or miR-451 coordinate with other gene products to dramatically alter the gene regulation capacities is also not known. Most likely, normal erythropoiesis and cell development of other tissues require dynamic regulation of miR-144/451 together with other genes at specific developmental stages or under particular physiological conditions. Addressing these questions will illustrate new general principles of miRNA biology. In this regard, comparison of the phenotypes in miR-144 and miR-451 single knockout mice with those of miR-144/451 double knockout animals upon various stresses or disease conditions will provide ample evidence for new functions of the miR-144/451 gene locus. Moreover, understanding how miR-144/451 impact disease development should identify new cellular pathways that are amenable to manipulation by standard pharmacologic approaches. In addition, miR-144 or miR-451 shows promise as diagnostic biomarkers of the onset, progression and prognosis of many diseases, as long as the contamination of erythrocytes when accessing the miR-144 or miR-451 levels is excluded. miR-144 and miR-451 are also emerge as potential therapeutic candidates, which could benefit for not only particular anemias [106, 29], but also non-erythroid diseases $[107,108]$. We conclude that miR-144 and miR-451, no matter the levels, regulate homeostasis and affect disease development both temporally and spatially.

\begin{abstract}
Abbreviations
5-FU: 5-fluorouracil; ABCA1: Adenosine triphosphate (ATP)-binding cassette transporter A1; AD: Alzheimer disease; ADAM: A disintegrin and metalloproteinase; Ago: Argonaute; AMPK: AMP-activated kinase; AP-1: Activator protein-1; BCL6: B-cell lymphoma 6; Cat: Catalase; ChIP: Chromatin immunoprecipitation; DGCR8: DiGeorge Syndrome Critical Region 8; DLBCL: Diffuse large B-cell lymphomas; EPO: Erythropoietin; FL: Fetal liver; FXR: Farnesoid X receptor; GATA1: GATA binding protein 1; GATA4: GATA binding protein 4; GBM: Glioblastoma multiforme; Gpx1: Glutathione peroxidase 1; HbS: Human hemoglobin S; HbSS: Homozygous sickle cell disease; HDL: High-density lipoprotein; HFD: High-fat diet; I/R: Ischemia/reperfusion; IPC: Ischemic preconditioning; klfd: Krüppel-like transcription factor d; MDR1: Multidrug resistance 1; MIF: Migration inhibitory factor; miRNA: microRNA; MO: Morpholino; mTOR: mammalian target of rapamycin; NSCLC: Non-small cell lung carcinoma; Oct1(POU2F1): Organic cation transporter 1 (POU domain, class 2 , transcription factor 1); PARN: Poly(A)-specific ribonuclease; Pax4: Paired box gene 4; PD: Parkinson's disease; PHZ: Phenohydrazine; pre-miRNA: Precursor microRNA; pri-miRNA: Primary microRNA; PTEN: Phosphatase and tensin homolog; PTU: 1phenyl-2-thiourea; RAB14: Ras-related protein Rab-14; RISC: RNA-induced silencing complex; ROS: Reactive oxygen species; shRNA: short-hairpin RNA; SMAD: Similar to mothers against decapentaplegic homolog; TSC1: Tuberous sclerosis complex 1; UTR: Untranslated region; WWHAZ: 14-3-3 protein zeta (14-3-36)
\end{abstract}

\section{Acknowledgments}

The authors appreciate Yuanjun Yu for language editing.

\section{Funding}

Several publications cited in this review were finished in our laboratory and this work is supported by the National Natural Science Foundation of China (81470277, 81670186 and 81870096 to DY).

Availability of data and materials Not applicable

\section{Authors' contributions}

TW and FW searched the literature and participated in the preparation of the manuscript. DY wrote the manuscript. All authors read and approved the final manuscript.

\section{Authors' information}

DY obtained his Ph.D. in Developmental Biology at the University of Wisconsin-Madison and finished his post-doctoral training at the University of Pennsylvania. DY has published over 50 peer-reviewed research articles including the ones listed as reference \#16, 19, 21, 30, 31, 40, 46, 64 and 77 in this review and now is the Director \& professor of the Jiangsu Key Laboratory of Experimental \& Translational Non-coding RNA Research at the Yangzhou University School of Medicine, Jiangsu Province, China.

Ethics approval and consent to participate Not applicable

Consent for publication

Not applicable

Competing interests

The authors declare that they have no competing interests. 


\section{Publisher's Note}

Springer Nature remains neutral with regard to jurisdictional claims in published maps and institutional affiliations.

\section{Author details \\ 'Jiangsu Key Laboratory of Experimental \& Translational Non-coding RNA Research, Yangzhou University School of Medicine, Yangzhou, China. ${ }^{2}$ Institute of Translational Medicine, Yangzhou University School of Medicine, Yangzhou, China. ${ }^{3}$ Jiangsu Co-Innovation Center for Prevention and Control of Important Animal Infectious Disease and Zoonosis, Yangzhou, China.}

\section{Received: 5 December 2018 Accepted: 15 March 2019}

\section{Published online: 07 May 2019}

\section{References}

1. Lee RC, Feinbaum RL, Ambros V. The C. elegans heterochronic gene lin-4 encodes small RNAs with antisense complementarity to lin-14. Cell. 1993;75: 843-54.

2. Bartel DP. Metazoan MicroRNAs. Cell. 2018;173:20-51.

3. Kim VN, Han J, Siomi MC. Biogenesis of small RNAs in animals. Nat Rev Mol Cell Biol. 2009;10:126-39.

4. Lee $\mathrm{Y}$, Jeon $\mathrm{K}$, Lee JT, Kim S, Kim VN. MicroRNA maturation: stepwise processing and subcellular localization. EMBO J. 2002;21:4663-70.

5. Lee Y, Ahn C, Han J, Choi H, Kim J, Yim J, et al. The nuclear RNase III Drosha initiates microRNA processing. Nature. 2003;425:415-9.

6. Nguyen TA, Jo MH, Choi YG, Park J, Kwon SC, Hohng S, et al. Functional anatomy of the human microprocessor. Cell. 2015;161:1374-87.

7. Hutvagner G, McLachlan J, Pasquinelli A, Balint E, Tuschl T, Zamore PD. A cellular function for the RNA-interference enzyme dicer in the maturation of the let-7 small temporal RNA. Science. 2001;293:834-8.

8. Grishok A, Pasquinelli A, Conte D, Li N, Parrish S, Ha I, et al. Genes and mechanisms related to RNA interference regulate expression of the small temporal RNAs that control C elegans developmental timing. Cell. 2001;106:23-34

9. Yang JS, Lai EC. Alternative miRNA biogenesis pathways and the interpretation of core miRNA pathway mutants. Mol Cell. 2011;43:892-903.

10. Okamura K, Hagen JW, Duan H, Tyler DM, Lai EC. The mirtron pathway generates microRNA-class regulatory RNAs in Drosophila. Cell. 2007;130:89-100.

11. Ruby JG, Jan CH, Bartel DP. Intronic microRNA precursors that bypass Drosha processing. Nature. 2007;448:83-6

12. Babiarz JE, Ruby JG, Wang Y, Bartel DP, Blelloch R. Mouse ES cells express endogenous shRNAs, siRNAs, and other microprocessor-independent, dicerdependent small RNAs. Genes Dev. 2008;22:2773-85

13. Flynt AS, Chung WJ, Greimann JC, Lima CD, Lai EC. microRNA biogenesis via splicing and exosome-mediated trimming in Drosophila. Mol Cell. 2010;38: 900-7.

14. Lai EC. microRNAs are complementary to $3^{\prime}$ UTR sequence motifs that mediate negative post-transcriptional regulation. Nat Genet. 2002:30:363-4.

15. Li J, Zhang Y. Current experimental strategies for intracellular target identification of microRNA. ExRNA. 2019:1:2-8.

16. Dore LC, Amigo JD, Dos Santos CO, Zhang Z, Gai X, Tobias JW, et al. A GATA-1-regulated microRNA locus essential for erythropoiesis. Proc Nat Acad Sci U S A. 2008:105:3333-8.

17. Zhang L, Flygare J, Wong P, Lim B, Lodish HF. miR-191 regulates mouse erythroblast enucleation by down-regulating Riok3 and Mxi1. Genes Dev. 2011;25:119-24.

18. Patrick DM, Zhang CC, Tao Y, Yao H, Qi X, Schwartz RJ, et al. Defective erythroid differentiation in miR-451 mutant mice mediated by 14-3-3zeta. Genes Dev. 2010:24:1614-9.

19. Yu D, Dos Santos CO, Zhao G, Jiang J, Amigo JD, Khandros E, et al. miR-451 protects against erythroid oxidant stress by repressing 14-3-3zeta. Genes Dev. 2010;24:1620-33.

20. Rasmussen KD, Simmini S, Abreu-Goodger C, Bartonicek N, Di Giacomo M, Bilbao-Cortes D, et al. The miR-144/451 locus is required for erythroid homeostasis. J Exp Med. 2010;207:1351-8.

21. Ding L, Zhang Y, Han L, Fu L, Mei X, Wang J, et al. Activating and sustaining c-Myc by depletion of miR-144/451 gene locus contributes to B-lymphomagenesis. Oncogene. 2018;37:1293-307.

22. Cheloufi S, Dos Santos CO, Chong MM, Hannon GJ. A dicer-independent miRNA biogenesis pathway that requires ago catalysis. Nature. 2010;465: 584-9.
23. Cifuentes D, Xue H, Taylor DW, Patnode H, Mishima Y, Cheloufi S, et al. A novel miRNA processing pathway independent of dicer requires Argonaute2 catalytic activity. Science. 2010;328:1694-8.

24. Yang JS, Maurin T, Robine N, Rasmussen KD, Jeffrey KL, Chandwani $R$, et al. Conserved vertebrate mir-451 provides a platform for dicer-independent, Ago2-mediated microRNA biogenesis. Proc Natl Acad Sci U S A. 2010;107: 15163-8.

25. Kloosterman WP, Wienholds E, de Bruijn E, Kauppinen S, Plasterk RH. In situ detection of miRNAs in animal embryos using LNA-modified oligonucleotide probes. Nat Methods. 2006;3(1):27-9.

26. Diez-Roux G, Banfi S, Sultan M, Geffers L, Anand S, Rozado D, et al. A highresolution anatomical atlas of the transcriptome in the mouse embryo. PLoS Biol. 2011:9(1):e1000582.

27. Fu YF, Du TT, Dong M, Zhu KY, Jing CB, Zhang Y, et al. Mir-144 selectively regulates embryonic a-hemoglobin synthesis during primitive erythropoiesis. Blood. 2008;113(6):1340-9.

28. Pase L, Layton JE, Kloosterman WP, Carradice D, Waterhouse PM, Lieschke GJ. miR-451 regulates zebrafish erythroid maturation in vivo via its target gata2. Blood. 2009:113(8):1794-804.

29. Zhan M, Miller CP, Papayannopoulou T, Stamatoyannopoulos G, Song CZ. MicroRNA expression dynamics during murine and human erythroid differentiation. Exp Hematol. 2007;35(7):1015-25.

30. Cheng $Y$, Wu W, Kumar SA, Yu D, Deng W, Tripic T, et al. Erythroid GATA1 function revealed by genome-wide analysis of transcription factor occupancy, histone modifications, and mRNA expression. Genome Res. 2009:19(12):2172-84.

31. Yu M, Riva L, Xie H, Schindler $Y$, Moran TB, Cheng $Y$, et al. Insights into GATA-1-mediated gene activation versus repression via genome-wide chromatin occupancy analysis. Mol Cell. 2009;36(4):682-95.

32. Wang $R$, Chen DQ, Huang JY, Zhang $K$, Feng $B$, Pan BZ, et al. Acquisition of radioresistance in docetaxel-resistant human lung adenocarcinoma cells is linked with dysregulation of miR-451/c-Myc-survivin/rad-51 signaling. Oncotarget. 2014;5:6113-29.

33. Bergamaschi A, Katzenellenbogen BS. Tamoxifen down-regulation of miR451 increases 14-3-3Z and promotes breast cancer cell survival and endocrine resistance. Oncogene. 2012;31:39-47.

34. Li X, Sanda T, Look AT, Novina CD, von Boehmer H. Repression of tumor suppressor miR-451 is essential for NOTCH1-induced oncogenesis in T-ALL. J Exp Med. 2011;208:663-75.

35. Du J, Liu S, He J, Liu X, Qu Y, Yan W, et al. MicroRNA-451 regulates stemness of side population cells via PI3K/Akt/mTOR signaling pathway in multiple myeloma. Oncotarget. 2015;6:14993-5007.

36. Su R, Gong JN, Chen MT, Song L, Shen C, Zhang XH, et al. C-Myc suppresses miR-451-YWHAZ/AKT axis via recruiting HDAC3 in acute myeloidleukemia. Oncotarget. 2016;7:77430-43.

37. Wang H, Wang A, Hu Z, Xu X, Liu Z, Wang Z. A critical role of miR-144 in diffuse large B-cell lymphoma proliferation and invasion. Cancer Immunol Res. 2016;4:337-44.

38. Soltani I, Douzi K, Gharbi $H$, Benhassine I, Teber $M$, Amouri $H$, et al. Downregulation of miR-451 in Tunisian chronic myeloid leukemia patients: potential implication in imatinib resistance. Hematology. 2017;22:201-7.

39. Dang CV. MYC on the path to cancer. Cell. 2012;149:22-35.

40. Chang TC, Yu D, Lee YS, Wentzel EA, Arking DE, West KM, et al. Widespread microRNA repression by Myc contributes to tumorigenesis. Nat Genet. 2008; 40:43-50

41. Miller DJ, Hayward DC, Reece-Hoyes JS, Scholten I, Catmull J, Gehring WJ, et al. Pax gene diversity in the basal cnidarian Acropora millepora (Cnidaria, Anthozoa): implications for the evolution of the Pax gene family. Proc Natl Acad Sci U S A. 2000;97:4475-80.

42. Zhang J, Qin X, Sun Q, Guo H, Wu X, Xie F, et al. Transcriptional control of PAX4-regulated miR-144/451 modulates metastasis by suppressing ADAMs expression. Oncogene. 2015;34(25):3283-95.

43. Zhang $X$, Wang $X$, Zhu H, Zhu C, Wang Y, Pu WT, et al. Synergistic effects of the GATA-4-mediated miR-144/451 cluster in protection against simulated ischemia/reperfusion-induced cardiomyocyte death. J Mol Cell Cardiol. 2010; 49:841-50.

44. Charron F, Nemer M. GATA transcription factors and cardiac development Semin Cell Dev Biol. 1999:10:85-91.

45. Ansari Kl, Ogawa D, Rooj AK, Lawler SE, Krichevsky AM, Johnson MD, et al. Glucose-based regulation of miR-451/AMPK signaling depends on the OCT1 transcription factor. Cell Rep. 2015;11(6):902-9. 
46. Fang X, Shen F, Lechauve C, Xu P, Zhao G, Itkow J, et al. miR-144/451 represses the LKB1/AMPK/mTOR pathway to promote red cell precursor survival during recovery from acute anemia. Haematologica. 2018;103(3): 406-16.

47. de Aguiar Vallim TQ, Tarling EJ, Kim T, Civelek M, Baldán Á, Esau C, et al. MicroRNA-144 regulates hepatic ATP binding cassette transporter A1 and plasma high-density lipoprotein after activation of the nuclear receptor Farnesoid X receptor. Circ Res. 2013;112:1603-12.

48. Gordon T, Castelli WP, Hjortland MC, Kannel WB, Dawber TR. High density lipoprotein as a protective factor against coronary heart disease: the Framingham study. Am J Med. 1977;62:707-14.

49. Tanzi RE, Bertram L. Twenty years of the Alzheimer's disease amyloid hypothesis. A genetic perspective. Cell. 2005;120:545-55

50. Persengiev S, Kondova I, Otting N, Koeppen AH, Bontrop RE. Genome-wide analysis of miRNA expression reveals a potential role for miR-144 in brain aging and spinocerebellar ataxia pathogenesis. Neurobiol Aging. 2011;32: 2316.e2317-27.

51. Cheng C, Li W, Zhang Z, Yoshimura S, Hao Q, Zhang C, et al. MicroRNA-144 is regulated by activator protein-1 (AP-1) and decreases expression of Alzheimer disease-related a disintegrin and metalloprotease 10 (ADAM10). J Biol Chem. 2013;288:13748-61.

52. Gal H, Pandi G, Kanner AA, Ram Z, Lithwick-Yanai G, Amariglio N, et al. MIR451 and imatinib mesylate inhibit tumor growth of glioblastoma stem cells. Biochem Biophys Res Commun. 2008;376:86-90.

53. Masaki S, Ohtsuka R, Abe Y, Muta K, Umemura T. Expression patterns of microRNAs 155 and 451 during normal human erythropoiesis. Biochem Biophys Res Commun. 2007;364:509-14.

54. Georgantas RW, Hildreth R, Morisot S, Alder J, Liu CG, Heimfeld S, et al. CD34+ hematopoietic stem-progenitor cell microRNA expression and function: a circuit diagram of differentiation control. Proc Natl Acad Sci U S A. 2007;104:2750-5.

55. Papapetrou EP, Korkola JE, Sadelain M. A genetic strategy for single and combinatorial analysis of miRNA function in mammalian hematopoietic stem cells. Stem Cells. 2009;28:287-96.

56. Du TT, Fu YF, Dong M, Wang L, Fan HB, Chen $Y$, et al. Experimental validation and complexity of miRNA-mRNA target interaction during zebrafish primitive erythropoiesis. Biochem Biophys Res Commun. 2009;381:688-93.

57. Sangokoya C, Telen MJ, Chi JT. microRNA miR-144 modulates oxidative stress tolerance and associates with anemia severity in sickle cell disease. Blood. 2010;116:4338-48.

58. LaMonte G, Philip N, Reardon J, Lacsina JR, Majoros W, Chapman L, et al. Translocation of sickle cell erythrocyte microRNAs into Plasmodium falciparum inhibits parasite translation and contributes to malaria resistance. Cell Host Microbe. 2012;12(2):187-99.

59. Ayi K, Turrini F, Piga A, Arese P. Enhanced phagocytosis of ringparasitized mutant erythrocytes: a common mechanism that may explain protection against falciparum malaria in sickle trait and beta- thalassemia trait. Blood. 2004;104:3364-71.

60. Williams TN, Mwangi TW, Roberts DJ, Alexander ND, Weatherall DJ, Wambua S, et al. An immune basis for malaria protection by the sickle cell trait. PLoS Med. 2005;2(5):e128.

61. Tiffert T, Lew VL, Ginsburg H, Krugliak M, Croisille L, Mohandas N. The hydration state of human red blood cells and their susceptibility to invasion by Plasmodium falciparum. Blood. 2005;105:4853-60.

62. Chen SY, Wang Y, Telen MJ, Chi JT. The genomic analysis of erythrocyte microRNA expression in sickle cell diseases. PLoS One. 2008;3:e2360.

63. Rathjen T, Nicol C, McConkey G, Dalmay T. Analysis of short RNAs in the malaria parasite and its red blood cell host. FEBS Lett. 2006;580:5185-8.

64. Wang W, Hang C, Zhang Y, Chen M, Meng X, Cao Q, et al. Dietary miR-45 protects erythroid cells from oxidative stress via increasing the activity of Foxo3 pathway. Oncotarget. 2017:8(63):107109-24.

65. Zhang L, Hou D, Chen X, Li D, Zhu L, Zhang Y, et al. Exogenous plant MIR168a specifically targets mammalian LDLRAP1: evidence of crosskingdom regulation by microRNA. Cell Res. 2012;22:107-26.

66. Chen D, Huang J, Zhang K, Pan B, Chen J, De W, et al. MicroRNA-451 induces epithelial-mesenchymal transition in docetaxel-resistant lung adenocarcinoma cells by targeting proto-oncogene c-Myc. Eur J Cancer. 2014;50(17):3050-67

67. Wang R, Wang ZX, Yang JS, Pan X, De W, Chen LB. MicroRNA-451 functions as a tumor suppressor in human non-small cell lung cancer by targeting ras-related protein 14 (RAB14). Oncogene. 2011;30(23):2644-58.
68. Bian HB, Pan X, Yang JS, Wang ZX, De W. Upregulation of microRNA-451 increases cisplatin sensitivity of non-small cell lung cancer cell line (A549). J Exp Clin Cancer Res. 2011;30(1):20.

69. van der Vos KE, Abels ER, Zhang X, Lai C, Carrizosa E, Oakley D, et al. Directly visualized glioblastoma-derived extracellular vesicles transfer RNA to microglia/macrophages in the brain. Neuro-Oncology. 2016;18(1):58-69.

70. Godlewski J, Nowicki MO, Bronisz A, Nuovo G, Palatini J, De Lay M, et al. MicroRNA-451 regulates LKB1/AMPK signaling and allows adaptation to metabolic stress in glioma cells. Mol Cell. 2010;37(5):620-32.

71. Nan $Y$, Han L, Zhang AL, Wang GX, Jia ZF, Yang Y, et al. MiRNA-451 plays a role as tumor suppressor in human glioma cells. Brain Res. 2010;1359:14-21.

72. Kovalchuk O, Filkowski J, Meservy J, Inntskyy Y, Tryndyak VP, Chekhun VF, et al. Involvement of microRNA-451 in resistance of the MCF-7 breast cancer cells to chemotherapeutic drug doxorubicin. Mol Cancer Ther. 2008;7(7):2152-9.

73. Iwaya T, Yokobori T, Nishida N, Kogo R, Sudo T, Tanaka F, et al. Downregulation of miR-144 is associated with colorectal cancer progression via activation of mTOR signaling pathway. Carcinogenesis. 2012:33(12):2391-7.

74. Bandres E, Bitarte N, Arias F, Agorreta J, Fortes P, Agirre X, et al. microRNA-451 regulates macrophage migration inhibitory factor production and proliferation of gastrointestinal cancer cells. Clin Cancer Res. 2009;15(7):2281-90.

75. Bitarte N, Bandres E, Boni V, Zarate R, Rodriquez J, Gonzalez-Huarriz M, et al. MicroRNA-451 is involved in the self-renewal, tumorigenicity, and chemoresistance of colorectal cancer stem cells. Stem Cells. 2011;29(11):1661-71.

76. Li Y, Wang J, Dai X, Zhou Z, Liu J, Zhang Y, et al. miR-451 regulates FoxO3 nuclear accumulation through Ywhaz in human colorectal cancer. Am J Transl Res. 2015;7:2775-85.

77. Zhang LY, Ho-Fun Lee V, Wong AM, Kwong DL, Zhu YH, Dong SS, et al. MicroRNA-144 promotes cell proliferation, migration and invasion in nasopharyngeal carcinoma through repression of PTEN. Carcinogenesis. 2013;34(2):454-63.

78. Liu N, Jiang N, Guo R, Jiang W, He Q, Xu Y, et al. MiR-451 inhibits cell growth and invasion by targeting MIF and is associated with survival in nasopharyngeal carcinoma. Mol Cancer. 2013;12:123.

79. Sureban SM, May R, Lightfoot SA, Hoskins AB, Lerner M, Brackett DJ, et al. DCAMKL-1 regulates epithelial-mesenchymal transition in human pancreatic cells through a miR-200a-dependent mechanism. Cancer Res. 2011;71(6):2328-38.

80. Jiang X, Shan A, Su Y, Cheng Y, Gu W, Wang W, et al. miR-144/451 promote cell proliferation via targeting PTEN/AKT pathway in insulinomas. Endocrinology. 2015;156(7):2429-39.

81. Gits C, van Kuijk P, Jonkers M, Boersma A, Smid M, van ljcken W, et al. MicroRNA expression profiles distinguish liposarcoma subtypes and implicate miR-145 and miR-451 as tumor suppressors. Int J Cancer. 2014:135:348348-61.

82. Zhu H, Wu H, Liu X, Evans BR, Medina DJ, Liu CG, et al. Role of microRNA miR-27a and miR-451 in the regulation of MDR1/P-glycoprotein expression in human cancer cells. Biochem Pharmacol. 2008;76:582-8.

83. Ribeiro-dos-Santos A, Khayat AS, Silva A, Alencar DO, Lobato J, Luz L, et al. Ultra-deep sequencing reveals the microRNA expression pattern of the human stomach. PLoS One. 2010;5:e13205.

84. Brenner B, Hoshen MB, Purim O, David MB, Ashkenazi K, Marshak G, et al. MicroRNAs as a potential prognostic factor in gastric cancer. World J Gastroenterol. 2011;17:3976-85.

85. Redova M, Poprach A, Nekvindova J, Iliev R, Radova L, Lakomy R, et al. Circulating miR-378 and miR-451 in serum are potential biomarkers for renal cell carcinoma. J Transl Med. 2012;10:55.

86. Ju X, Li D, Shi Q, Hou H, Sun N, Shen B. Differential microRNA expression in childhood B-cell precursor acute lymphoblastic leukemia. Pediatr Hematol Oncol. 2009;26:1-10.

87. Jones KB, Salah Z, Del Mare S, Galasso M, Gaudio E, Nuovo GJ, et al. miRNA signatures associate with pathogenesis and progression of osteosarcoma. Cancer Res. 2012:72:1865-77.

88. Godlewski J, Bronisz A, Nowicki MO, Chiocca EA, Lawler S. Micro-RNA-451: a conditional switch controlling glioma cell proliferation and migration. Cell Cycle. 2010;9:2742-8.

89. Wang $X$, Zhu H, Zhang X, Liu Y, Chen J, Medvedovic M, et al. Loss of the miR-144/451 cluster impairs ischemic preconditioning-mediated cardioprotection by targeting Rac-1. Cardiovasc Res. 2012;94:379-90.

90. Kuwabara Y, Horie T, Baba O, Watanabe S, Nishiga M, Usami S, et al. MicroRNA-451 exacerbates lipotoxicity in cardiac myocytes and high-fat diet-induced cardiac hypertrophy in mice through suppression of the LKB1/AMPK pathway. Circ Res. 2015;116(2):279-88. 
91. Zhuo S, Yang M, Zhao Y, Chen X, Zhang F, Li N, et al. microRNA-451 negatively regulates hepatic glucose production and glucose homeostasis by targeting glycerol kinase mediated gluconeogenesis. Diabetes. 2016;65(11): 3276-88.

92. Karp CM, Tan TT, Mathew R, Nelson D, Mukherjee C, Degenhardt K, et al. Role of the polarity determinant crumbs in suppressing mammalian epithelial tumor progression. Cancer Res. 2008;68:4105-15.

93. Tsuchiya S, Oku M, Imanaka $Y$, Kunimoto $R$, Okuno $Y$, Terasawa $K$, et al. MicroRNA-338-3p and microRNA-451 contribute to the formation of basolateral polarity in epithelial cells. Nucl Acids Res. 2009;37:3821-7.

94. Murata K, Yoshitomi H, Furu M, Ishikawa M, Shibuya $\mathrm{H}$, Ito H, et al. MicroRNA-451 down-regulates neutrophil chemotaxis via p38 MAPK. Arthritis Rheumatol. 2014;66(3):549-59.

95. Jelkmann W. Physiology and pharmacology of erythropoietin. Transfus Med Hemother. 2013:40(5):302-9.

96. Ogunshola OO, Bogdanova AY. Epo and non-hematopoietic cells: what do we know? Methods Mol Biol. 2013;982:13-41.

97. Alural B, Duran GA, Tufekci KU, Allmer J, Onkal Z, Tunali D, et al. EPO mediates neurotrophic, neuroprotective, anti-oxidant, and anti-apoptotic effects via downregulation of miR-451 and miR-885-5p in SH-SY5Y neuron-like cells. Front Immunol. 2014;5:475.

98. Ramírez CM, Rotllan N, Vlassov AV, Dávalos A, Li M, Goedeke L, et al. Control of cholesterol metabolism and plasma high-density lipoprotein levels by microRNA-144. Circ Res. 2013:112:1592-601.

99. Rosenberger CM, Podyminogin RL, Diercks AH, Treuting PM, Peschon JJ, Rodriguez D, et al. miR-144 attenuates the host response to influenza virus by targeting the TRAF6-IRF7 signaling axis. PLoS Pathog. 2017;13(4): e1006305.

100. Murphy CP, Li X, Maurer V, Oberhauser M, Gstir R, Wearick-Silva LE, et al. MicroRNA-mediated rescue of fear extinction memory by miR-144-3p in extinction-impaired miceMiR-144-3p mediated rescue of impaired fear extinction. Biol Psychiatry. 2017;81(12):979-89.

101. Yang J, Maurin T, Lai EC. Functional parameters of dicer-independent microRNA biogenesis. RNA. 2012;18:945-57.

102. Weill L, Belloc E, Bava FA, Mendez R. Translational control by changes in poly(a) tail length: recycling mRNAs. Nat Struct Mol Biol. 2012;19:577-85.

103. Yoda M, Cifuentes D, Izumi N, Sakaguchi Y, Suzuki T, Giraldez AJ, et al. PARN mediates 3 '-end trimming of Argonaute2-cleaved precursor microRNAs. Cell Rep. 2013;5(3):715-26.

104. Liu J, Carmell MA, Rivas FV, Marsden CG, Thomson JM, Song JJ, et al. Argonaute2 is the catalytic engine of mammalian RNAi. Science. 2004; 305:1437-41.

105. Yi T, Arthanari H, Akabayov B, Song H, Papadopoulos E, Qi HH, et al. elF1A augments Ago2-mediated dicer-independent miRNA biogenesis and RNA interference. Nat Commun. 2015;6:7194.

106. Li B, Zhu X, Ward CM, Starlard-Davenport A, Takezaki M, Berry A, et al. MIR144-mediated NRF2 gene silencing inhibits fetal hemoglobin expression in sickle cell disease. Exp Hematol. 2019;70:85-96.

107. Chen DQ, Yu C, Zhang XF, Liu ZF, Wang R, Jiang M, et al. HDAC3-mediated silencing of miR-451 decreases chemosensitivity of patients with metastaticcastration-resistant prostate cancer by targeting NEDD9. Ther Adv Med Oncol. 2018;10:1-15.

108. Pan X, Wang R, Wang ZX. The potential role of miR-451 in cancer diagnosis, prognosis, and therapy. Mol Cancer Ther. 2013;12(7):1153-62.

\section{Ready to submit your research? Choose BMC and benefit from:}

- fast, convenient online submission

- thorough peer review by experienced researchers in your field

- rapid publication on acceptance

- support for research data, including large and complex data types

- gold Open Access which fosters wider collaboration and increased citations

- maximum visibility for your research: over $100 \mathrm{M}$ website views per year

At BMC, research is always in progress.

Learn more biomedcentral.com/submissions 